\title{
Bose gases, Bose-Einstein condensation, and the Bogoliubov approximation
}

\author{
Robert Seiringer ${ }^{\text {a) }}$ \\ IST Austria, Am Campus 1, 3400 Klosterneuburg, Austria
}

(Received 15 October 2013; accepted 2 April 2014; published online 26 June 2014)

\begin{abstract}
We review recent progress towards a rigorous understanding of the Bogoliubov approximation for bosonic quantum many-body systems. We focus, in particular, on the excitation spectrum of a Bose gas in the mean-field (Hartree) limit. A list of open problems will be discussed at the end. (C) 2014 AIP Publishing LLC. [http://dx.doi.org/10.1063/1.4881536]
\end{abstract}

\section{INTRODUCTION}

Bose-Einstein condensation (BEC) in cold atomic gases was first experimentally realized in 1995. ${ }^{1,8}$ In these experiments, a large number of (bosonic) atoms are confined to a trap and cooled to very low temperatures. Below a critical temperature condensation of a large fraction of particles into the same one-particle state occurs.

Various interesting quantum phenomena have been explored in these and subsequent experiments, like the appearance of quantized vortices in rotating systems and the related property of superfluidity. The latter is related to the low-energy excitation spectrum of the system. We refer to Refs. 2,5,7, and 13 for reviews of the recent developments in this field.

BEC was predicted by Einstein in $1924,{ }^{12}$ building upon a previous derivation of Planck's formula for black-body radiation by Bose. ${ }^{4}$ Einstein's considerations were based on an ideal (i.e., non-interacting) Bose gas. The presence of particle interactions represents a major difficulty for a rigorous derivation of this phenomenon, however. One of the key contributions to the theory of weakly interacting Bose gases is Bogoliubov's 1947 paper, ${ }^{3}$ where he introduces an approximate model (now referred to as the Bogoliubov approximation) to explain its superfluid behavior. In this paper, we will summarize recent progress made towards a rigorous justification of this approximation.

\section{THE BOSE GAS: A QUANTUM MANY-BODY PROBLEM}

The quantum-mechanical description of a system of $N$ bosons is given in terms of the Hamiltonian, acting as a linear operator in a suitable Hilbert space. For bosons interacting via a pair-interaction potential denoted by $v(x)$, it is given, in appropriate units, by

$$
H_{N}=-\sum_{i=1}^{N} \Delta_{i}+\sum_{1 \leq i<j \leq N} v\left(x_{i}-x_{j}\right) .
$$

The kinetic energy is described by $\Delta$, the Laplacian on a suitable domain in $\mathbb{R}^{3}$, which we will typically take to be a cube of side length $L$, i.e., $[0, L]^{3}$. Suitable boundary conditions have to be imposed, with periodic boundary conditions being a typical example. The subscript $i$ indicates, as usual, that the second derivative is with respect to $x_{i} \in \mathbb{R}^{3}$.

As appropriate for bosons, the Hamiltonian $H_{N}$ acts on the Hilbert space of permutationsymmetric wave functions $\Psi\left(x_{1}, \ldots, x_{N}\right)$ in $\bigotimes^{N} L^{2}\left([0, L]^{3}\right)$. The interaction $v$ is assumed to be short-range, i.e., it decays fast enough to be integrable at infinity, and mostly repulsive to ensure that

\footnotetext{
a)Email: robert.seiringer@ist.ac.at
} 
the system behaves like a gas at low density and low temperature. A particularly simple example is the special case of hard spheres of diameter $a>0$, where, formally, $v(x)=\infty$ for $|x| \leq a$, and $v(x)=0$ for $|x|>a$.

The following quantities, derived from the Hamiltonian $H_{N}$, will interest us here.

- Ground state energy, defined as the lowest value of the spectrum of the Hamiltonian,

$$
E_{0}(N, L)=\inf \operatorname{spec} H_{N} .
$$

For large systems, one can take a thermodynamic limit $N \rightarrow \infty, L \rightarrow \infty$ with $N / L^{3}=\varrho$ fixed, and consequently define the ground state energy density as

$$
e(\varrho)=\lim _{L \rightarrow \infty} \frac{E_{0}\left(\varrho L^{3}, L\right)}{L^{3}} .
$$

- At positive temperature $T=\beta^{-1}>0$, one considers instead the free energy

$$
F(N, L, T)=-\frac{1}{\beta} \ln \operatorname{Tr} \exp \left(-\beta H_{N}\right),
$$

and the corresponding free energy density in the thermodynamic limit

$$
f(\varrho, T)=\lim _{L \rightarrow \infty} \frac{F\left(\varrho L^{3}, L, T\right)}{L^{3}} .
$$

- The ground state wave function $\Psi_{0}$, being a function of $N \gg 1$ variables, is for all practical purposes too complicated to compute. Instead one considers the corresponding reduced density matrices of $\Psi_{0}$, the simplest of which is the one-particle density matrix, given by the integral kernel

$$
\gamma_{0}\left(x, x^{\prime}\right)=N \int_{\mathbb{R}^{3(N-1)}} \Psi_{0}\left(x, x_{2}, \ldots, x_{N}\right) \overline{\Psi_{0}\left(x^{\prime}, x_{2}, \ldots, x_{N}\right)} d x_{2} \cdots d x_{N} .
$$

It satisfies $0 \leq \gamma_{0} \leq N$ as an operator, and $\operatorname{Tr} \gamma_{0}=N$. With the aid of creation and annihilation operators (to be reviewed in Sec. IV below) one can also write

$$
\gamma_{0}\left(x, x^{\prime}\right)=\left\langle a^{\dagger}\left(x^{\prime}\right) a(x)\right\rangle
$$

and this definition generalizes to arbitrary mixed states as well.

- The diagonal of the one-particle density matrix is the particle density

$$
\varrho_{0}(x)=\gamma_{0}(x, x)=N \int_{\mathbb{R}^{3(N-1)}}\left|\Psi_{0}\left(x, x_{2}, \ldots, x_{N}\right)\right|^{2} d x_{2} \cdots d x_{N},
$$

with $\int \varrho_{0}(x) d x=N$. For translation invariant systems in the thermodynamic limit, $\varrho_{0}$ is a constant and does not depend on $x$, but for inhomogeneous systems the spatial variation of $\varrho_{0}$ represents a non-trivial question.

- By definition, Bose-Einstein condensation in a state $\Psi_{0}$ means that the one-particle density matrix $\gamma_{0}$ has an eigenvalue of order $N$, i.e., that $\left\|\gamma_{0}\right\|_{\infty} \geq c N$ for some $c>0$ and all (large) $N$. The corresponding eigenfunction is called the condensate wave function. BEC is expected to occur below a critical temperature.

For translation invariant systems with Hamiltonian of the form (1) one always has

$$
\left\|\gamma_{0}\right\|_{\infty}=\frac{1}{L^{3}} \int_{[0, L]^{6}} \gamma_{0}\left(x, x^{\prime}\right) d x d x^{\prime}
$$

in the ground state, or any Gibbs state at positive temperature. This being of the order $N=\varrho L^{3}$ means that $\gamma_{0}\left(x, x^{\prime}\right)$ does not decay as $\left|x-x^{\prime}\right| \rightarrow \infty$, a property which is also termed long range order.

- Of particular interest to us will be the structure of the excitation spectrum, i.e., the spectrum of $H_{N}$ above the ground state energy $E_{0}(N)$, and the relation of the corresponding eigenstates 
to the ground state. For translation invariant systems, $H_{N}$ commutes with the total momentum operator

$$
P=-i \sum_{j=1}^{N} \nabla_{j},
$$

and hence one can look at their joint spectrum. Of particular relevance is the infimum

$$
E_{q}(N, L)=\inf \operatorname{spec} H_{N} \uparrow_{P=q},
$$

and one can investigate the limit

$$
e_{q}(\varrho)=\lim _{L \rightarrow \infty}\left(E_{q}\left(\varrho L^{3}, L\right)-E_{0}\left(\varrho L^{3}, L\right)\right)
$$

for fixed $\varrho$ and $q$. In contrast to the non-interacting case, where $e_{q}(\varrho)=0$ for all $q$ and $\varrho$, one expects a linear behavior of $e_{q}(\varrho)$ for small $q$ for interacting particles. For a review of various questions related to the excitation spectrum of Bose gases we refer to Ref. 6.

\section{THE IDEAL BOSE GAS}

For non-interacting bosons, i.e., in the case $v \equiv 0$, the free energy can be calculated explicitly in terms of its Legendre transform. It is given by

$$
f_{0}(\varrho, T)=\sup _{\mu \leq 0}\left[\mu \varrho+\frac{1}{(2 \pi)^{3} \beta} \int_{\mathbb{R}^{3}} \ln \left(1-\exp \left(-\beta\left(p^{2}-\mu\right)\right)\right) d p\right],
$$

and satisfies the simple scaling relation

$$
f_{0}(\varrho, T)=\varrho^{5 / 3} f_{0}\left(\beta \varrho^{2 / 3}, 1\right) .
$$

From (13) one can immediately infer the following property: If $\varrho$ exceeds a certain critical density, namely, if

$$
\varrho \geq \varrho_{c}(\beta) \equiv \frac{1}{(2 \pi)^{3}} \int_{\mathbb{R}^{3}} \frac{1}{e^{\beta p^{2}}-1} d p=\left(\frac{T}{4 \pi}\right)^{3 / 2} \zeta(3 / 2),
$$

(where $\zeta$ denotes the Riemann zeta function) the supremum in (13) is achieved at $\mu=0$, and hence $\partial f_{0} / \partial \varrho=0$ for $\varrho \geq \varrho_{c}$. That is, the free energy becomes independent of the density above the critical density. Because of the scaling relation (14) one can equivalently talk about a critical temperature, which equals

$$
T_{c}(\varrho)=\frac{4 \pi}{\zeta(3 / 2)^{2 / 3}} \varrho^{2 / 3} .
$$

The one-particle density matrix for the ideal Bose gas can also be calculated explicitly, and is given by

$$
\gamma_{0}(x, y)=\left[\varrho-\varrho_{c}(\beta)\right]_{+}+\sum_{n \geq 0} \frac{e^{\beta \mu_{e} n}}{(4 \pi \beta n)^{3 / 2}} e^{-|x-y|^{2} /(4 \beta n)} .
$$

Here, $[\cdot]_{+}=\max \{0, \cdot\}$ denotes the positive part of a real number, and $\mu_{\varrho} \leq 0$ is the $\mu$ where the supremum is achieved in (13). In particular, $\mu_{\varrho}=0$ for $T \leq T_{c}$. The last sum in (17) can easily be seen to decay exponentially in $|x-y|$ if $\mu_{\varrho}<0$, while it decays algebraically (like $|x-y|^{-1}$, in fact) for $\mu_{\varrho}=0$. We thus conclude the following asymptotic behavior of $\gamma_{0}$ depending on the temperature:

- For $T<T_{c}$ or, equivalently, $\varrho>\varrho_{c}, \gamma_{0}$ does not decay at infinity, but converges to the positive number $\varrho-\varrho_{c}$, which equals the condensate density.

- For $T>T_{c}, \gamma_{0}$ decays exponentially in $|x-y|$.

- At the critical value $T=T_{c}, \gamma_{0}$ decays algebraically. 
These features of the ideal Bose gas are expected to be correct even in the presence of interparticle interactions, with a different value of the critical temperature $T_{c}$, but so far no general result of this kind is known. In the case of purely repulsive interactions, one can show that at large enough temperature there is always exponential decay. ${ }^{40}$ That is, one can derive an upper bound on the critical temperature in this case, but no (non-zero) lower bounds are available to this date.

The ground state energy of the ideal Bose gas is of course identically zero, and also the excitation spectrum can easily be computed explicitly. It is simply given by all finite sums of the form

$$
\sum_{p \neq 0} p^{2} n_{p}
$$

where the sum is over $p \in\left(\frac{2 \pi}{L} \mathbb{Z}\right)^{3}$ and $n_{p} \in\{0,1,2, \ldots\}$ for each $p$. In particular, one easily checks that $e_{q}(\varrho)$ defined in (12) is identically zero for all $q \in \mathbb{R}^{3}$.

\section{SECOND QUANTIZATION ON FOCK SPACE}

In the following, it will be convenient to regard $\bigotimes_{\mathrm{sym}}^{N} L^{2}\left([0, L]^{3}\right)$ as a subspace of the bosonic Fock space

$$
\mathcal{F}=\bigoplus_{n=0}^{\infty} \bigotimes_{\text {sym }}^{n} L^{2}\left([0, L]^{3}\right)
$$

On this space, the particle number $N$ is now an operator, which acts simply as multiplication by $n$ on the subspace $\bigotimes_{\text {sym }}^{n} L^{2}\left([0, L]^{3}\right)$.

A basis of $L^{2}\left([0, L]^{3}\right)$ is given by the plane waves $L^{-3 / 2} e^{i p \cdot x}$ for $p \in\left(\frac{2 \pi}{L} \mathbb{Z}\right)^{3}$, and we introduce the corresponding creation and annihilation operators, which satisfy the canonical commutation relations $(\mathrm{CCR})$

$$
\left[a_{p}, a_{q}\right]=\left[a_{p}^{\dagger}, a_{q}^{\dagger}\right]=0, \quad\left[a_{p}, a_{q}^{\dagger}\right]=\delta_{p, q} .
$$

The Hamiltonian $H_{N}$ is equal to the restriction of

$$
\mathbb{H}=\sum_{p}|p|^{2} a_{p}^{\dagger} a_{p}+\frac{1}{2 L^{3}} \sum_{p} \widehat{v}(p) \sum_{q, k} a_{q+p}^{\dagger} a_{k-p}^{\dagger} a_{k} a_{q}
$$

to the subspace $\bigotimes_{\text {sym }}^{N} L^{2}\left([0, L]^{3}\right) \subset \mathcal{F}$, where

$$
\widehat{v}(p)=\int_{[0, L]^{3}} v(x) e^{-i p x} d x
$$

denotes the Fourier transform of $v$.

\section{THE BOGOLIUBOV APPROXIMATION}

At low energy, and for sufficiently weak interactions, one expects the occurrence of BoseEinstein condensation. That is, the zero momentum mode is expected to be macroscopically occupied, meaning that $a_{0}^{\dagger} a_{0} \sim N$. In particular, the $p=0$ mode plays a special role.

The Bogoliubov approximation consists of

- dropping all terms in $\mathbb{H}$ higher than quadratic in $a_{p}^{\dagger}$ and $a_{p}$ for $p \neq 0$,

- replacing $a_{0}^{\dagger}$ and $a_{0}$ in $\mathbb{H}$ by $\sqrt{N}$.

The resulting Hamiltonian is quadratic in the $a_{p}^{\dagger}$ and $a_{p}$, and equals (note that the contribution of $p=0$ to the second sum in (21) is exactly equal to $N(N-1) \widehat{v}(0) /\left(2 L^{3}\right)$, hence the substitution of 
$a_{0}^{\dagger}$ and $a_{0}$ by $\sqrt{N}$ was not applied to this term)

$$
\begin{aligned}
\mathbb{H}^{\mathrm{Bog}}= & \frac{N(N-1)}{2 L^{3}} \widehat{v}(0) \\
& +\sum_{p \neq 0}\left(\left(|p|^{2}+\varrho \widehat{v}(p)\right) a_{p}^{\dagger} a_{p}+\frac{1}{2} \varrho \widehat{v}(p)\left(a_{p}^{\dagger} a_{-p}^{\dagger}+a_{p} a_{-p}\right)\right),
\end{aligned}
$$

with $\varrho=N / L^{3}$ the particle density. It can be explicitly diagonalized via a Bogoliubov transformation:

Let $b_{p}=\cosh \left(\alpha_{p}\right) a_{p}+\sinh \left(\alpha_{p}\right) a_{-p}^{\dagger}$, with

$$
\tanh \left(\alpha_{p}\right)=\frac{|p|^{2}+\varrho \widehat{v}(p)-\sqrt{|p|^{4}+2|p|^{2} \varrho \widehat{v}(p)}}{\varrho \widehat{v}(p)} .
$$

Here, we have to assume that $|p|^{2}+2 \varrho \widehat{v}(p) \geq 0$ for all $p$ in order for the square root to be welldefined. The $b_{p}$ and $b_{p}^{\dagger}$ again satisfy CCR (for any choice of real numbers $\alpha_{p}$, in fact). A simple calculation shows that

$$
\mathbb{H}^{\mathrm{Bog}}=E_{0}^{\mathrm{Bog}}+\sum_{p \neq 0} e_{p} b_{p}^{\dagger} b_{p}
$$

where

$$
E_{0}^{\mathrm{Bog}}=\frac{N(N-1)}{2 L^{3}} \widehat{v}(0)-\frac{1}{2} \sum_{p \neq 0}\left(|p|^{2}+\varrho \widehat{v}(p)-\sqrt{|p|^{4}+2|p|^{2} \varrho \widehat{v}(p)}\right)
$$

and

$$
e_{p}=\sqrt{|p|^{4}+2|p|^{2} \varrho \widehat{v}(p)}
$$

Note that in contrast to the non-interacting case, where $e_{p}=p^{2}$, the function $e_{p}$ in (27) behaves linearly in $p$ for small $p$ (assuming that $\widehat{v}$ does not vanish near zero, i.e., that $\widehat{v}(0)>0$ ).

The Bogoliubov approximation thus predicts that the ground state energy density in the thermodynamic limit equals

$$
e^{\operatorname{Bog}}(\varrho)=\frac{1}{2} \varrho^{2} \widehat{v}(0)-\frac{1}{2(2 \pi)^{3}} \int_{\mathbb{R}^{3}}\left(|p|^{2}+\varrho \widehat{v}(p)-\sqrt{|p|^{4}+2|p|^{2} \varrho \widehat{v}(p)}\right) d p .
$$

For small $\varrho$, it turns out that

$$
\begin{aligned}
e^{\mathrm{Bog}}(\varrho)= & \frac{1}{2} \varrho^{2}\left(\widehat{v}(0)-\frac{1}{2(2 \pi)^{3}} \int_{\mathbb{R}^{3}} \frac{|\widehat{v}(p)|^{2}}{|p|^{2}} d p\right) \\
& +4 \pi \frac{128}{15 \sqrt{\pi}}\left(\frac{\varrho \widehat{v}(0)}{8 \pi}\right)^{5 / 2}+o\left(\varrho^{5 / 2}\right),
\end{aligned}
$$

where the numeric factor in the last term arises from the integral

$$
\frac{128}{15 \sqrt{\pi}}=-\sqrt{\frac{8}{\pi^{3}}} \int_{\mathbb{R}^{3}}\left(|p|^{2}+1-\sqrt{|p|^{4}+2|p|^{2}}-\frac{1}{2|p|^{2}}\right) d p .
$$

The expression $\widehat{v}(0)-\frac{1}{2(2 \pi)^{3}} \int_{\mathbb{R}^{3}} \frac{|\widehat{v}(p)|^{2}}{|p|^{2}} d p$ can be identified with the first two terms in the Born series for $8 \pi a$, where $a$ denotes the scattering length of $v$. The latter can, e.g., be defined as ${ }^{18}$

$$
a=\frac{1}{8 \pi}\left\langle|v|^{1 / 2}\left|\frac{1}{1+\frac{1}{2} v^{1 / 2} \frac{1}{p^{2}}|v|^{1 / 2}}\right| v^{1 / 2}\right\rangle
$$

whenever the operator in question is invertible, i.e., whenever $p^{2}+\frac{1}{2} v$ does not have a zero-energy resonance. Here, $v^{1 / 2}$ is defined as $v|v|^{-1 / 2}$ if $v \neq 0$, and as zero otherwise. 
Since the scattering length is the relevant physical parameter at low energy, this suggests that the true ground state energy density for small $\varrho$ should be

$$
e(\varrho)=4 \pi a \varrho^{2}\left(1+\frac{128}{15 \sqrt{\pi}} \sqrt{\varrho a^{3}}+o\left(\varrho^{1 / 2}\right)\right) .
$$

This expression for $e(\varrho)$ is known as the Lee-Huang-Yang formula. ${ }^{21}$ Its rigorous justification is one of the open problems discussed below in Sec. VIII.

Not only does the Bogoliubov approximation make a prediction about the ground state energy of the system, it also allows to compute the complete excitation spectrum. In fact, from (25) we see that the spectrum of $\mathbb{H}^{\mathrm{Bog}}-E_{0}^{\mathrm{Bog}}$ is given by

$$
\sum_{p} e_{p} n_{p} \quad \text { with } n_{p} \in\{0,1,2, \ldots\},
$$

with $e_{p}$ defined in (27). Moreover, the corresponding eigenstates can be constructed out of the ground state $\Psi_{0}$ by elementary excitations of the form

$$
b_{p_{n}}^{\dagger} \cdots b_{p_{1}}^{\dagger} \Psi_{0},
$$

with $b_{p}^{\dagger}=\cosh \left(\alpha_{p}\right) a_{p}^{\dagger}+\sinh \left(\alpha_{p}\right) a_{-p}$, as before.

One can also calculate the ground state energy $E_{q}^{\mathrm{Bog}}$ in a sector of total momentum $q$, and arrives at

$$
\begin{aligned}
e_{q}(\varrho)=\lim _{L \rightarrow \infty}\left(E_{q}^{\mathrm{Bog}}-E_{0}^{\mathrm{Bog}}\right) & =\text { subadditive hull of } e_{p} \\
& =\inf _{\sum_{p} p n_{p}=q} \sum_{p} e_{p} n_{p} .
\end{aligned}
$$

In particular, also $e_{q}(\varrho)$ behaves linearly in $q$ for small $q$.

For a detailed discussion of variants of the Bogoliubov approximation we refer the interested reader to Ref. 46.

\section{VALIDITY OF THE BOGOLIUBOV APPROXIMATION}

There are only very few rigorous results concerning the validity of the Bogoliubov approximation:

- Quite generally, one can show that the pressure in the thermodynamic limit is unaffected by the substitution of $a_{0}^{\dagger}$ and $a_{0}$ (or any other mode) by complex numbers (called $c$-numbers in the physics literature), see Refs. 15, 29, and 42. This is true independent of whether or not BEC occurs in the system. Moreover, the value of the occupation number $a_{0}^{\dagger} a_{0}$ is correctly predicted, to leading order in the system size, by the approximate model with $a_{0}^{\dagger}$ and $a_{0}$ replaced by $c$-numbers.

- The Lieb-Liniger model of one-dimensional bosons with $\delta$-function interaction is defined by the Hamiltonian

$$
H_{N}=\sum_{j=1}^{N}-\frac{\partial^{2}}{\partial z_{j}^{2}}+g \sum_{1 \leq i<j \leq N} \delta\left(z_{i}-z_{j}\right)
$$

on $\bigotimes_{\text {sym }}^{N} L^{2}([0, L])$. As shown in Ref. 25, this model is exactly solvable, and various quantities, like the ground state energy density and the excitation spectrum, can be computed. As far as the ground state energy is concerned, the Bogoliubov approximation turns out to become exact in the weak coupling/high density limit $g / \varrho \rightarrow 0$. The same is true for parts of the excitation spectrum, but the exact excitation spectrum has an additional branch (called the Lieb-mode) which is absent in the Bogoliubov approximation. 
- For charged bosons in a uniform background (also known as the "jellium" model) Foldy's law $^{14}$

$$
e(\varrho) \approx C \varrho^{5 / 4}
$$

for the ground state energy density has been verified in Refs. 30 and 41. Also in this case, the Bogoliubov approximation becomes exact in the high density limit, i.e., as $\varrho \rightarrow \infty$. In this model, the interaction among the particles is given by the Coulomb potential $v(x)=|x|^{-1}$. For charge neutrality, an additional one-particle potential $\sum_{i=1}^{N} V\left(x_{i}\right)$ of the form

$$
V(x)=-\int_{[0, L]^{3}} \frac{\varrho}{|x-y|} d y
$$

is added, as well as a constant to take into account the self-interaction of the background, $\frac{1}{2} \varrho^{2} \iint_{[0, L]^{6}}|x-y|^{-1} d x d y$. The total energy per unit volume then satisfies (37) with an explicit constant $C$ given by the integral

$$
\begin{aligned}
C & =-\frac{1}{2(2 \pi)^{3}} \int_{\mathbb{R}^{3}}\left(|p|^{2}+\frac{4 \pi}{|p|^{2}}-\sqrt{|p|^{4}+8 \pi}\right) d p \\
& =-\frac{2}{5} \frac{\Gamma(3 / 4)}{\Gamma(5 / 4)}\left(\frac{2}{\pi}\right)^{1 / 4},
\end{aligned}
$$

which arises from the Bogoliubov approximation in a similar fashion as discussed in Sec. V. There is also a corresponding result for a two-component charged Bose gas, where the validity of the Bogoliubov approximation for the ground state energy was rigorously verified. ${ }^{31,41}$

- The leading term in the ground state energy of the low density Bose gas,

$$
e(\varrho) \approx 4 \pi a \varrho^{2},
$$

is rigorously known to be correct for purely repulsive interaction potentials, i.e., for $v \geq 0$, with finite scattering length. An upper bound of the correct form was provided by Dyson already in $1957,{ }^{10}$ while a proof of the lower bound was given only in 1998 by Lieb and Yngvason. ${ }^{33}$ For generalizations to partially attractive interactions see Refs. 20 and 44, and for a corresponding result for the free energy at positive temperature see Refs. 37 and 45 . We emphasize that these results do not, strictly speaking, concern a rigorous justification of the Bogoliubov approximation, since the latter does not actually predict the correct appearance of the scattering length, but only yields its first two orders in Born approximation, as discussed in Sec. V.

An upper bound on the ground state energy density of the conjectured form of the Lee-HuangYang formula

$$
4 \pi a \varrho^{2}\left(1+\frac{128}{15 \sqrt{\pi}} \sqrt{\varrho a^{3}}+o\left(\varrho^{1 / 2}\right)\right)
$$

was proved in Ref. 43 for positive, smooth, and sufficiently weak interaction potentials. A proof of a lower bound of this form is still an outstanding open problem.

- For low density $\varrho$, the Bogoliubov approximation can only be strictly valid if

- the third term in the Born series for the scattering length is negligible,

- the second term in the Born series for the scattering length is large compared with $a\left(a^{3} \varrho\right)^{1 / 2}$.

Consider an interaction potential of the form

$$
\frac{a_{0}}{R^{3}} v(x / R)
$$

for "nice" $v$ with $\int v=8 \pi$, and $R>0$ an adjustable parameter, which is allowed to depend on the density of the system, and will be chosen large compared to $a_{0}$. Then $a \approx a_{0}$ to leading order. In terms of $R$, the two conditions above are equivalent to

$$
\frac{a^{3}}{R^{2}} \ll a\left(a^{3} \varrho\right)^{1 / 2} \ll \frac{a^{2}}{R}
$$


If we write $a / R \sim\left(a^{3} \varrho\right)^{1 / 2-\delta}$ for small $a^{3} \varrho$, this requires $\delta$ to satisfy $0<\delta<1 / 4$. Note that for this choice of $R$ one has $R^{3} \varrho \sim\left(a^{3} \varrho\right)^{3 \delta-1 / 2}$. In particular, the range $R$ of the interaction potential is much smaller than the mean particle spacing $\varrho^{-1 / 3}$ only if $\delta>1 / 6$.

In Ref. 16, the validity of the Lee-Huang-Yang formula (41) is proved for interaction potentials of the form (42) and small enough $\delta$. An extension of this result to a larger range of $\delta$, including $\delta$ slightly larger than $1 / 6$, was announced in Ref. 32 .

Most of these results concern the validity of the Bogoliubov approximation for the ground state energy of the system. Much less is known as far as the excitation spectrum is concerned. The only case where the Bogoliubov approximation can be rigorously justified even for the excitation spectrum concerns a system in the mean-field (or Hartree) limit, where the interactions among the particles are very weak and of long range. These results are fairly recent and we review them in Sec. VII.

\section{THE MEAN-FIELD (HARTREE) LIMIT}

A simpler case where the analysis of the validity of the Bogoliubov approximation can be extended beyond the ground state energy is the Hartree limit. This is an extreme form of a meanfield limit where the interaction potential extends over the whole size of the system, but the interaction is sufficiently weak (of order $1 / N$ ) in order for the interaction energy to be of the same order as the kinetic energy.

We consider again a system of $N$ bosons in a cubic box, with periodic boundary conditions. For simplicity, let us choose units such that the length of the box $L$ equals 1 . The Hamiltonian of the systems is thus given by

$$
H_{N}=-\sum_{i=1}^{N} \Delta_{i}+\frac{1}{N-1} \sum_{1 \leq i<j \leq N} v\left(x_{i}-x_{j}\right) .
$$

Here we wrote the interaction potential as $(N-1)^{-1} v(x)$, reflecting the weakness of the potential as mentioned above. The case of fixed, $N$-independent $v$ corresponds to the mean-field or Hartree limit.

It is not difficult to see that the ground state energy is determined, to leading order in $N$ for large $N$, by minimizing the energy $\left\langle\Psi\left|H_{N}\right| \Psi\right\rangle$ over product states of the form

$$
\Psi\left(x_{1}, \ldots, x_{N}\right)=\phi\left(x_{1}\right) \cdots \phi\left(x_{N}\right) .
$$

This has been shown, in a much more general setting than what is discussed here, in Ref. 22. For a constant $\phi$, corresponding to a homogeneous system, the resulting Hartree energy is then simply equal to $\frac{1}{2} N \int v$.

It is also known that starting from a product state of the form (45), a solution to the timedependent Schrödinger equation $i \partial_{t} \Psi=H_{N} \Psi$ stays roughly a product at later times, with the factors in the limit $N \rightarrow \infty$ determined by the time-dependent Hartree equation

$$
i \partial_{t} \phi=-\Delta \phi+\left(|\phi|^{2} * v\right) \phi,
$$

where $*$ denotes convolution. For a history of this problem and a review of recent results, we refer to Ref. 34.

Going beyond the leading order, where the Hartree equation applies, we can ask the following questions.

- Given that the ground state energy $E_{0}(N)=\inf \operatorname{spec} H_{N}$ satisfies $E_{0}(N)=\frac{1}{2} N \widehat{v}(0)+o(N)$ for fixed (i.e., $N$-independent) $v$, what is the next order correction? It turns out that it is actually $O(1)$, and the $O(1)$-term can be explicitly computed and agrees with the prediction from the Bogoliubov approximation.

- What is the spectrum of $H_{N}-E_{0}(N)$, i.e., the excitation spectrum of the system? Does it converge as $N \rightarrow \infty$ ? Is the Bogoliubov approximation valid? The latter predicts a dispersion law for elementary excitations that is linear for small momentum, as discussed in Sec. V. 
- What fraction of particles is in a Bose-Einstein condensate? Recall that Bose-Einstein condensation concerns the largest eigenvalue of the one-particle density matrix $\gamma$ of a many-body wave function $\Psi$, defined via the matrix elements

$$
\begin{aligned}
& \langle f|\gamma| g\rangle \\
& =N \int \overline{f(x)} \Psi\left(x, x_{2}, \ldots, x_{N}\right) g(y) \overline{\Psi\left(y, x_{2}, \ldots, x_{N}\right)} d x d y d x_{2} \cdots d x_{N} .
\end{aligned}
$$

For fixed $v$, the Bogoliubov approximation predicts that $\|\gamma\| \geq N-O(1)$ in the ground state, and this can actually be proved to be correct.

\section{A. Main results}

For our analysis of the excitation spectrum, we assume that $v(x)$ is bounded and of positive type, i.e.,

$$
v(x)=\sum_{p \in(2 \pi \mathbb{Z})^{3}} \widehat{v}(p) e^{i p \cdot x} \quad \text { with } \widehat{v}(p) \geq 0 \forall p \in(2 \pi \mathbb{Z})^{3} .
$$

Under these assumptions, the following theorem holds.

Theorem 1. The ground state energy $E_{0}(N)$ of $H_{N}$ equals

$$
E_{0}(N)=\frac{N}{2} \widehat{v}(0)+E_{0}^{\mathrm{Bog}}+O\left(N^{-1 / 2}\right)
$$

with

$$
E_{0}^{\text {Bog }}=-\frac{1}{2} \sum_{p \neq 0}\left(|p|^{2}+\widehat{v}(p)-\sqrt{|p|^{4}+2|p|^{2} \widehat{v}(p)}\right) .
$$

Moreover, the excitation spectrum of $H_{N}-E_{0}(N)$ below an energy $\xi$ is equal to finite sums of the form

$$
\sum_{p \in(2 \pi \mathbb{Z})^{3} \backslash\{0\}} e_{p} n_{p}+O\left(\xi^{3 / 2} N^{-1 / 2}\right),
$$

where

$$
e_{p}=\sqrt{|p|^{4}+2|p|^{2} \widehat{v}(p)},
$$

and $n_{p} \in\{0,1,2, \ldots\}$ for all $p \neq 0$.

Theorem 1 is proved in Ref. 38. The proof consists of constructing a unitary operator $U$ that makes $U H_{N} U^{\dagger}$ close to the operator:

$$
\frac{N}{2} \widehat{v}(0)+E_{0}^{\mathrm{Bog}}+\sum_{p \in(2 \pi \mathbb{Z})^{3} \backslash\{0\}} e_{p} a_{p}^{\dagger} a_{p} .
$$

In particular, the proof implies that the excited eigenfunctions can be (approximately) obtained by acting with products of $U^{\dagger} a_{p}^{\dagger} a_{0} U$ on the ground state.

Let us comment on the error terms in (49) and (51). Both the ground state energy and all excited energy levels a distance $O(1)$ from the ground state agree with the prediction obtained via Bogoliubov's approximation up to errors of order $N^{-1 / 2}$ for large $N$. Moreover, an excitation energy a distance $\xi$ from the ground state energy is necessarily of the form $\sum_{p} e_{p} n_{p}(1+o(1))$ as long as $\xi^{3 / 2} N^{-1 / 2} \ll \xi$, i.e., for $\xi \ll N$. That is, the Bogoliubov approximation gives the correct excitation energies to leading order in a very large window above the ground state energy, whose size has to be small compared with $N$. This restriction is presumably optimal. The existence of Bose-Einstein condensation is only guaranteed for excitation energies small compared to $N$, and the existence of $\mathrm{BEC}$ is one of the key assumptions entering the Bogoliubov approximation. 


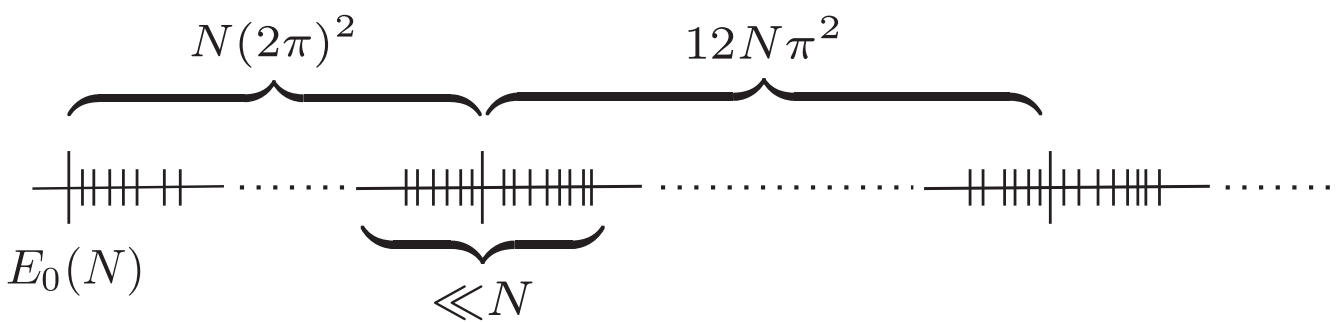

FIG. 1. Sketch of the parts of the spectrum that are correctly determined by the Bogoliubov approximation in the Hartree limit.

Theorem 1 implies the following corollary concerning the momentum dependence of the spectrum of $H_{N}$.

Corollary 1. Let $E_{P}(N)$ denote the ground state energy of $H_{N}$ in the sector of total momentum $P$. We have

$$
E_{P}(N)-E_{0}(N)=\min _{\left\{n_{p}\right\}, \sum_{p} p n_{p}=P} \sum_{p \neq 0} e_{p} n_{p}+O\left(|P|^{3 / 2} N^{-1 / 2}\right) .
$$

In particular,

$$
E_{P}(N)-E_{0}(N) \geq|P| \min _{p} \sqrt{2 \widehat{v}(p)+|p|^{2}}+O\left(|P|^{3 / 2} N^{-1 / 2}\right) .
$$

The bound (55) implies that $E_{P}(N)-E_{0}(N)$ behaves linearly in $P$ for not too large $P$ (assuming that $\widehat{v}(p)$ does not vanish for small $p$ ). Note that this fact is caused by the interactions among the particles, non-interacting systems do not show this behavior. The linear behavior is very important physically and is responsible for the superfluid behavior of the system. According to Landau, the coefficient in front of $|P|$ is, in fact, the critical velocity for frictionless flow. We refer to Ref. 6 for further details on this correspondence.

Note that under the unitary transformation

$$
\widetilde{U}=\exp \left(-i q \cdot \sum_{j=1}^{N} x_{j}\right), \quad q \in(2 \pi \mathbb{Z})^{3},
$$

the Hamiltonian $H_{N}$ transforms as

$$
\widetilde{U}^{\dagger} H_{N} \widetilde{U}=H_{N}+N|q|^{2}-2 q \cdot P,
$$

where $P=-i \sum_{j=1}^{N} \nabla_{j}$ denotes again the total momentum operator. Hence our results apply equally also to the parts of the spectrum of $H_{N}$ with excitation energies close to $N|q|^{2}$, corresponding to collective excitations where the particles move uniformly with momentum $q$; cf. Fig. 1 .

\section{B. Ideas in the proof}

In the language of second quantization, the Hamiltonian $H_{N}$ is the restriction of the operator

$$
\mathbb{H}=\sum_{p \in(2 \pi \mathbb{Z})^{3}}|p|^{2} a_{p}^{\dagger} a_{p}+\frac{1}{2(N-1)} \sum_{p} \widehat{v}(p) \sum_{q, k} a_{q+p}^{\dagger} a_{k-p}^{\dagger} a_{k} a_{q}
$$

to the $N$-particle subspace of the Fock space $\mathcal{F}$. Note that $N$ has two different roles here. It determines the particle number, but also appears as a parameter in the Hamiltonian $\mathbb{H}$.

As discussed in Sec. V, the Bogoliubov approximation consists of

- dropping all terms higher than quadratic in $a_{p}^{\dagger}$ and $a_{p}, p \neq 0$;

- replacing $a_{0}^{\dagger}$ and $a_{0}$ by $\sqrt{N}$. 
The resulting quadratic Hamiltonian is $\frac{N}{2} \widehat{v}(0)+\mathbb{H}^{\mathrm{Bog}}$, where

$$
\mathbb{H}^{\mathrm{Bog}}=\sum_{p \neq 0}\left(\left(|p|^{2}+\widehat{v}(p)\right) a_{p}^{\dagger} a_{p}+\frac{1}{2} \widehat{v}(p)\left(a_{p}^{\dagger} a_{-p}^{\dagger}+a_{p} a_{-p}\right)\right) .
$$

It is diagonalized via a Bogoliubov transformation of the form

$$
b_{p}=\cosh \left(\alpha_{p}\right) a_{p}+\sinh \left(\alpha_{p}\right) a_{-p}^{\dagger},
$$

leading to

$$
\mathbb{H}^{\mathrm{Bog}}=E_{0}^{\mathrm{Bog}}+\sum_{p \neq 0} e_{p} b_{p}^{\dagger} b_{p}
$$

with $E_{0}^{\text {Bog }}$ and $e_{p}$ defined in (50) and (52), respectively.

The proof of Theorem 1 consists of two main steps:

1. As a first step, one shows that $H_{N}$ is well approximated by an operator similar to the Bogoliubov Hamiltonian $\mathbb{H}^{\text {Bog }}$, but with $a_{p}$ and $a_{p}^{\dagger}$ replaced by

$$
a_{p}^{\dagger} \rightarrow c_{p}^{\dagger}:=\frac{a_{p}^{\dagger} a_{0}}{\sqrt{N}}, \quad a_{p} \rightarrow c_{p}:=\frac{a_{p} a_{0}^{\dagger}}{\sqrt{N}} .
$$

Note that the operators $c_{p}$ and $c_{p}^{\dagger}$ conserve the particle number. The resulting Hamiltonian is quadratic in $c_{p}^{\dagger}$ and $c_{p}$ and is, in particular, also particle number conserving. Hence, it has a chance of being close to $H_{N}$ on the subspace of particle number $N$. The original Bogoliubov Hamiltonian (59) does not leave this subspace invariant, and hence can not be directly compared with $H_{N}$.

2. Mimicking the Bogoliubov transformation (60), we introduce the operators $d_{p}=\cosh \left(\alpha_{p}\right) c_{p}+$ $\sinh \left(\alpha_{p}\right) c_{-p}^{\dagger}$. It turns out that the modified Hamiltonian from Step 1 is close to

$$
E_{0}^{\mathrm{Bog}}+\sum_{p \neq 0} e_{p} d_{p}^{\dagger} d_{p}
$$

whose spectrum now has to be analyzed. This analysis is complicated by the fact that the operators $d_{p}$ and $d_{p}^{\dagger}$ do not satisfy CCR. It turns out that they do, however, approximately on the subspace where $a_{0}^{\dagger} a_{0}$ is close to $N$, which is sufficient for our purpose.

In the following, we shall explain these two steps in greater detail. For further details, we refer to Ref. 38.

\section{Step 1: Approximation by a quadratic Hamiltonian}

Under our assumptions on the interaction potential $v$, it is not difficult to see that

$$
N-a_{0}^{\dagger} a_{0} \leq \text { const. }\left[1+H_{N}-E_{0}(N)\right] .
$$

This proves that the excitation energy dominates the condensate depletion. In particular, if the excitation energy is small compared with $N$, most particles occupy the zero momentum mode, i.e., Bose-Einstein condensation occurs.

To show that cubic and quartic terms in $a_{p}^{\dagger}$ and $a_{p}, p \neq 0$, in the Hamiltonian are negligible, one needs to prove a stronger bound of the form

$$
\left(N-a_{0}^{\dagger} a_{0}\right)^{2} \leq \text { const. }\left[1+\left(H_{N}-E_{0}(N)\right)^{2}\right]
$$

however. It implies that also the fluctuations in the number of particles outside the condensate are suitably small. 
The first statement (64) follows easily from positivity of $\widehat{v}(p)$. Positivity implies that

$$
\sum_{p \in(2 \pi \mathbb{Z})^{3} \backslash\{0\}} \widehat{v}(p)\left|\sum_{j=1}^{N} e^{i p \cdot x_{j}}\right|^{2} \geq 0,
$$

which can be rewritten as

$$
\sum_{1 \leq i<j \leq N} v\left(x_{i}-x_{j}\right) \geq \frac{N^{2}}{2} \widehat{v}(0)-\frac{N}{2} v(0) .
$$

Thus, $H_{N}$ is bounded from below as

$$
H_{N} \geq \frac{N}{2} \widehat{v}(0)+T-\frac{N}{2(N-1)}(v(0)-\widehat{v}(0)),
$$

where $T$ denotes the kinetic energy

$$
T=-\sum_{i=1}^{N} \Delta_{i}
$$

The statement (64) follows, since $T \geq(2 \pi)^{2}\left(N-a_{0}^{\dagger} a_{0}\right)$.

For the second statement (65) one has to work a bit more. It turns out to be useful to actually prove a slightly stronger bound, namely, the inequality

$$
\left(N-a_{0}^{\dagger} a_{0}\right) T \leq \text { const. }\left[1+\left(H_{N}-E_{0}(N)\right)^{2}\right] .
$$

Since $T \geq(2 \pi)^{2}\left(N-a_{0}^{\dagger} a_{0}\right)$ (and the two operators commute), this indeed implies the bound (65).

For the proof of (70), let us introduce the notation

$$
N^{>}=N-a_{0}^{\dagger} a_{0}=\sum_{i=1}^{N} Q_{i}
$$

for the number of particles outside the condensate, where $Q$ denotes the projection onto the subspace of $L^{2}\left([0,1]^{3}\right)$ of co-dimension one orthogonal to the constant function. For any bosonic (i.e., permutation-symmetric) wave function $\Psi$, we can write

$$
\begin{aligned}
\left\langle\Psi\left|N^{>} T\right| \Psi\right\rangle & =N\left\langle\Psi\left|Q_{1} T\right| \Psi\right\rangle \\
& =N\left\langle\Psi\left|Q_{1} S\right| \Psi\right\rangle+\left\langle\Psi\left|N^{>}\left(H_{N}-E_{0}(N)\right)\right| \Psi\right\rangle,
\end{aligned}
$$

where

$$
\begin{aligned}
S & =T-H_{N}+E_{0}(N) \\
& =E_{0}(N)-(N-1)^{-1} \sum_{i<j} v\left(x_{i}-x_{j}\right) .
\end{aligned}
$$

Using Schwarz's inequality, the last term in (72) can be bounded as

$$
\left\langle\Psi\left|N^{>}\left(H_{N}-E_{0}(N)\right)\right| \Psi\right\rangle \leq\left\langle\Psi\left|\left(N^{>}\right)^{2}\right| \Psi\right\rangle^{1 / 2}\left\langle\Psi\left|\left(H_{N}-E_{0}(N)\right)^{2}\right| \Psi\right\rangle^{1 / 2} .
$$

We split $S$ into two parts, $S=S_{a}+S_{b}$, with

$$
S_{a}=E_{0}(N)-\frac{1}{N-1} \sum_{2 \leq i<j \leq N} v\left(x_{i}-x_{j}\right)
$$

and

$$
S_{b}=-\frac{1}{N-1} \sum_{j=2}^{N} v\left(x_{1}-x_{j}\right)
$$


Note that $S_{a}$ does not depend on $x_{1}$. Using the positivity of $\widehat{v}(p)$ as in (66), but with the sum over $j$ running from 2 to $N$ only, as well as the simple upper bound $E_{0}(N) \leq \frac{N}{2} \widehat{v}(0)$ on the ground state energy, we see that

$$
S_{a} \leq \frac{1}{2}(\widehat{v}(0)+v(0))
$$

In particular, this implies that

$$
N\left\langle\Psi\left|Q_{1} S_{a}\right| \Psi\right\rangle \leq \frac{1}{2}(\widehat{v}(0)+v(0))\left\langle\Psi\left|N^{>}\right| \Psi\right\rangle
$$

To bound the contribution of $S_{b}$, we use

$$
\begin{aligned}
-\left\langle\Psi\left|Q_{1} S_{b}\right| \Psi\right\rangle=\left\langle\Psi\left|Q_{1} v\left(x_{1}-x_{2}\right)\right| \Psi\right\rangle= & \left\langle\Psi\left|Q_{1} Q_{2} v\left(x_{1}-x_{2}\right)\right| \Psi\right\rangle \\
& +\left\langle\Psi\left|Q_{1} P_{2} v\left(x_{1}-x_{2}\right) P_{2}\right| \Psi\right\rangle \\
& +\left\langle\Psi\left|Q_{1} P_{2} v\left(x_{1}-x_{2}\right) Q_{2}\right| \Psi\right\rangle,
\end{aligned}
$$

where $P=1-Q$ denotes the rank-one projection onto the constant function in $L^{2}\left([0,1]^{3}\right)$. The second term on the right side of (79) is positive. For the first and the third, we use Schwarz's inequality and $\|v\|_{\infty}=v(0)$ to conclude that

$$
\left\langle\Psi\left|Q_{1} S_{b}\right| \Psi\right\rangle \leq v(0)\left\langle\Psi\left|Q_{1} Q_{2}\right| \Psi\right\rangle^{1 / 2}+v(0)\left\langle\Psi\left|Q_{1}\right| \Psi\right\rangle
$$

Since

$$
\left\langle\Psi\left|Q_{1} Q_{2}\right| \Psi\right\rangle=\frac{\left\langle\Psi\left|N^{>}\left(N^{>}-1\right)\right| \Psi\right\rangle}{N(N-1)} \leq \frac{\left\langle\Psi\left|\left(N^{>}\right)^{2}\right| \Psi\right\rangle}{N^{2}},
$$

we have thus shown that

$$
\begin{aligned}
\left\langle\Psi\left|N^{>} T\right| \Psi\right\rangle \leq & \frac{1}{2}(\widehat{v}(0)+3 v(0))\left\langle\Psi\left|N^{>}\right| \Psi\right\rangle \\
& +\left(v(0)+\left\langle\Psi\left|\left(H_{N}-E_{0}(N)\right)^{2}\right| \Psi\right\rangle^{1 / 2}\right)\left\langle\Psi\left|\left(N^{>}\right)^{2}\right| \Psi\right\rangle^{1 / 2}
\end{aligned}
$$

Using that $N^{>} \leq(2 \pi)^{-2} T$ in the last factor, this further implies that

$$
\begin{aligned}
\left\langle\Psi\left|N^{>} T\right| \Psi\right\rangle \leq & \left(\frac{v(0)+\left\langle\Psi\left|\left(H_{N}-E_{0}(N)\right)^{2}\right| \Psi\right\rangle^{1 / 2}}{2 \pi}\right)^{2} \\
& +(3 v(0)+\widehat{v}(0))\left\langle\Psi\left|N^{>}\right| \Psi\right\rangle
\end{aligned}
$$

The desired result (70) then follows from (64).

\section{An algebraic identity} by

The inequalities (64) and (70) allow us to conclude that $\mathbb{H}$ is, at low energy, well approximated

$$
\frac{N}{2} \widehat{v}(0)+\frac{1}{2} \sum_{p \neq 0}\left[A_{p}\left(c_{p}^{\dagger} c_{p}+c_{-p}^{\dagger} c_{-p}\right)+B_{p}\left(c_{p}^{\dagger} c_{-p}^{\dagger}+c_{p} c_{-p}\right)\right]
$$


where $A_{p}=|p|^{2}+\widehat{v}(p)$ and $B_{p}=\widehat{v}(p)$, and the operators $c_{p}$ are defined in (62). A simple identity, which does not use the CCR, is

$$
\begin{aligned}
& A_{p}\left(c_{p}^{\dagger} c_{p}+c_{-p}^{\dagger} c_{-p}\right)+B_{p}\left(c_{p}^{\dagger} c_{-p}^{\dagger}+c_{p} c_{-p}\right) \\
& =\sqrt{A_{p}^{2}-B_{p}^{2}}\left(\frac{\left(c_{p}^{\dagger}+\beta_{p} c_{-p}\right)\left(c_{p}+\beta_{p} c_{-p}^{\dagger}\right)}{1-\beta_{p}^{2}}+\frac{\left(c_{-p}^{\dagger}+\beta_{p} c_{p}\right)\left(c_{-p}+\beta_{p} c_{p}^{\dagger}\right)}{1-\beta_{p}^{2}}\right) \\
& -\frac{1}{2}\left(A_{p}-\sqrt{A_{p}^{2}-B_{p}^{2}}\right)\left(\left[c_{p}, c_{p}^{\dagger}\right]+\left[c_{-p}, c_{-p}^{\dagger}\right]\right),
\end{aligned}
$$

where

$$
\beta_{p}= \begin{cases}\frac{1}{B_{p}}\left(A_{p}-\sqrt{A_{p}^{2}-B_{p}^{2}}\right) & \text { if } B_{p}>0 \\ 0 & \text { if } B_{p}=0\end{cases}
$$

Note that if the operators $c_{p}$ and $c_{p}^{\dagger}$ satisfied CCR, the term in the last line of (85) would be a constant. Its deviation from a constant can be controlled in terms of the condensate depletion, and the inequality (70) can be used to control the error made by simply replacing it be the value it would take in the case of CCR.

Introducing the operators

$$
d_{p}=\frac{c_{p}+\beta_{p} c_{-p}^{\dagger}}{\sqrt{1-\beta_{p}^{2}}}
$$

and their adjoints, we conclude that $\mathbb{H}$ is, in fact, close to the operator

$$
\frac{N}{2} \widehat{v}(0)+E_{0}^{\mathrm{Bog}}+\sum_{p \neq 0} e_{p} d_{p}^{\dagger} d_{p}
$$

where we used that

$$
E_{0}^{\mathrm{Bog}}=-\frac{1}{2} \sum_{p \neq 0}\left(A_{p}-\sqrt{A_{p}^{2}-B_{p}^{2}}\right)
$$

and

$$
e_{p}=\sqrt{A_{p}^{2}-B_{p}^{2}}
$$

\section{Step 2: The spectrum of $d_{p}^{\dagger} d_{p}$}

If the operators $d_{p}$ and $d_{p}^{\dagger}$ satisfied CCR, we could immediately read off the spectrum of the operator in (88), and we would be done. However, without CCR we do not know the spectrum of $d_{p}^{\dagger} d_{p}$ and, moreover, the various summands in (88) do not actually commute in our case.

The usual Bogoliubov transformation (60) is of the form

$$
b_{p}=\cosh \left(\alpha_{p}\right) a_{p}+\sinh \left(\alpha_{p}\right) a_{-p}^{\dagger}=e^{-X} a_{p} e^{X},
$$

where $X$ is the anti-hermitian operator

$$
X=\frac{1}{2} \sum_{p \neq 0} \alpha_{p}\left(a_{p}^{\dagger} a_{-p}^{\dagger}-a_{p} a_{-p}\right) .
$$

This identity can easily be verified using the CCR $\left[a_{p}, a_{q}^{\dagger}\right]=\delta_{p, q}$. Our operators $c_{p}=a_{p} a_{0}^{\dagger} / \sqrt{N}$, on the other hand, satisfy

$$
\left[c_{p}, c_{q}^{\dagger}\right]=\delta_{p, q} \frac{a_{0} a_{0}^{\dagger}}{N}-\frac{a_{p} a_{q}^{\dagger}}{N} .
$$


We now define, in analogy to (92), the particle-number conserving anti-hermitian operator

$$
\widetilde{X}=\frac{1}{2} \sum_{p \neq 0} \alpha_{p}\left(c_{p}^{\dagger} c_{-p}^{\dagger}-c_{p} c_{-p}\right) .
$$

In order to compute the spectrum of $d_{p}^{\dagger} d_{p}$, we apply the unitary $e^{\widetilde{X}}$, and argue that the resulting operator is close $a_{p}^{\dagger} a_{p}$, at least in the subspace of low energy. More precisely, we show that

$$
e^{-\widetilde{X}} a_{p} e^{\widetilde{X}}=\overbrace{\cosh \left(\alpha_{p}\right) c_{p}+\sinh \left(\alpha_{p}\right) c_{-p}^{\dagger}}^{d_{p}}+\text { Error }_{p}
$$

for suitable small error term. Here it is important that actually the sum over all error terms (depending on $p$ ) is still (relatively) small as long as $\left(N-a_{0}^{\dagger} a_{0}\right)^{2} \ll N^{2}$. The proof of (95) is somewhat lengthy and will be skipped here. It proceeds by studying $e^{-t \widetilde{X}} a_{p} e^{t \widetilde{X}}$ as a function of $t \in[0,1]$, using a Grönwall type estimate. The details are presented in Ref. 38 .

\section{Conclusions and generalizations}

The mean-field or Hartree limit may be somewhat unphysical when it comes to the description of cold atomic gases. It can be used as a toy model, however, which is analytically much easier to handle than the Gross-Pitaevskii limit of dilute gases, ${ }^{26-28,39}$ for instance. The results reviewed in this section are the first rigorous results concerning the excitation spectrum of an interacting Bose gas, in a suitable limit of weak, long-range interactions. With the notable exception of exactly solvable models in one dimension, this is the only model where rigorous results on the excitation spectrum are available. The results verify Bogoliubov's prediction that the spectrum consists of sums of elementary excitations. In the translation invariant case, the excitation energy turns out to be linear in the momentum for small momentum. In particular, Landau's criterion for superfluidity is verified.

The methods presented in this section can be generalized to inhomogeneous systems without translation invariance. This was shown in Ref. 17, where the excitation spectrum of the Hamiltonian

$$
H_{N}=\sum_{i=1}^{N}\left(-\Delta_{i}+V\left(x_{i}\right)\right)+\frac{1}{N-1} \sum_{1 \leq i<j \leq N} v\left(x_{i}-x_{j}\right)
$$

on the Hilbert space $\otimes_{\mathrm{sym}}^{N} L^{2}\left(\mathbb{R}^{3}\right)$ was studied, with a trap potential $V$ that is locally bounded and tends to infinity at infinity, in order to ensure that all the particles are confined and cannot escape to infinity. To leading order in $N$, the ground state energy of (96) is determined by minimizing the Hartree functional

$$
\begin{aligned}
\mathcal{E}^{\mathrm{H}}(\phi)= & \int_{\mathbb{R}^{3}}\left(|\nabla \phi(x)|^{2}+V(x)|\phi(x)|^{2}\right) d x \\
& +\frac{1}{2} \int_{\mathbb{R}^{6}}|\phi(x)|^{2} v(x-y)|\phi(y)|^{2} d x d y,
\end{aligned}
$$

with minimal energy $E^{\mathrm{H}}=\inf \left\{\mathcal{E}^{\mathrm{H}}(\phi): \int|\phi|^{2}=1\right\}$. Under the stated conditions on $v$ and $V$, it is not difficult to see that there exists a unique minimizer $\phi_{0}$ (up to a constant phase, of course, which we can choose such that $\phi_{0}$ is positive) with $E^{\mathrm{H}}=\mathcal{E}^{\mathrm{H}}\left(\phi_{0}\right)$. The corresponding Euler-Lagrange equation for the minimizer $\phi_{0}$ can be written as $K^{\mathrm{H}} \phi_{0}=0$, where $K^{\mathrm{H}}$ is the Hartree operator

$$
K^{\mathrm{H}}=-\Delta+V(x)+v *\left|\phi_{0}\right|^{2}(x)-\varepsilon_{0},
$$

with $\varepsilon_{0}=E^{\mathrm{H}}+\frac{1}{2} \int_{\mathbb{R}^{6}}\left|\phi_{0}(x)\right|^{2} v(x-y)\left|\phi_{0}(y)\right|^{2} d x d y$ and $*$ denoting convolution.

The excitation spectrum of (96) turns out to have a similar structure as in (51), i.e., it consists of sums of elementary excitations. These are described by an effective one-body operator given by

$$
E=\left(\sqrt{K^{\mathrm{H}}}\left(K^{\mathrm{H}}+2 W\right) \sqrt{K^{\mathrm{H}}}\right)^{1 / 2}
$$


where $W$ denotes the operator with integral kernel $\phi_{0}(x) v(x-y) \phi_{0}(y)$. More precisely, to leading order in $N$ the spectrum of $H_{N}-E_{0}(N)$ is of the form $\sum_{i} e_{i} n_{i}$, with $n_{i} \in\{0,1,2, \ldots\}$ and $e_{i}$ the (non-zero) eigenvalues of $E$. We refer to Ref. 17 for details.

By using different techniques, this result was further generalized in Ref. 24, where the validity of the Bogoliubov approximation in the Hartree limit was shown for a much larger class of Hamiltonians and interaction potentials. The method of Ref. 24 does not require that $v$ has positive Fourier transform, for instance, one merely needs to assume that the corresponding Hartree functional has a unique minimizer and that the Hessian is strictly positive at the minimum. While the result of Ref. 24 applies to a much larger class of models, it does not yield so precise estimates on the error terms as the ones obtained in Theorem 1, and is restricted to studying the excitation spectrum in a window of order one above the ground state energy.

It remains to be seen to what extent the methods in Refs. 17 and 38 or the method in Ref. 24 can be generalized to the study of less restrictive parameter regimes, away from the Hartree limit. A first step in this direction was recently taken in Ref. 9, where bounds were given on the maximally allowed rate at which the system size is allowed to grow with $N$ in order for the Bogoliubov approximation to remain valid. Equivalently, one can let the interaction potential $v$ depend on $N$ and ask at what rate it is allowed to tend to a $\delta$-function as $N \rightarrow \infty$. Since all error terms in Theorem 1 are explicit, an estimate of this kind is actually contained in Theorem 1, but the dependence of these error terms on $v$ was greatly improved in Ref. 9.

Finally, we mention that the validity of the Bogoliubov approximation in the Hartree limit can also be investigated concerning the dynamics generated by the Hamiltonian $H_{N}$. We refer to Ref. 23 and the references there for recent results in this direction.

\section{OPEN PROBLEMS}

In this final section, we collect a list of open problems related to the Bogoliubov approximation for many-boson systems. Some of these problems have already been mentioned in Secs. VI and VII.

- One of the key assumptions motivating the Bogoliubov approximation is the existence of Bose-Einstein condensation. While this property is easy to demonstrate in the Hartree limit discussed in Sec. VII, it is not known how to prove it in more general cases. In particular, the existence of BEC in the usual thermodynamic limit remains an open problem. The only model where the occurrence of BEC has been proved in the thermodynamic limit is the hard-core lattice gas at exactly half-filling, which is equivalent to the quantum XY spin model. ${ }^{11,19}$ BEC is also known to occur in the Gross-Pitaevskii limit of dilute trapped gases. ${ }^{26-28,39}$

- As discussed in Sec. VI, the ground state energy density of a dilute Bose gas with repulsive interactions is known to equal $4 \pi a \varrho^{2}$ to leading order in $\varrho$, with $a$ denoting the scattering length of the interaction potential. The first correction term to this expression is expected to be of the form of the Lee-Huang-Yang formula displayed in Eq. (41) above. In particular, also this correction term is expected to depend on the interaction $v$ only via its scattering length. It is an interesting open problem to establish the formula (41) rigorously, i.e., to give a suitable lower bound on the ground state energy that agrees with (41) up to terms of lower order. As discussed in Sec. VI, an upper bound of the correct form was recently derived in Ref. 43, at least for smooth and suitably small interaction potentials such that the Born series for the scattering length converges. In the more general case, even an upper bound of the right form is unknown.

- The results in Sec. VII on the excitation spectrum concern the mean-field or Hartree limit, where the interaction among the particles is very weak and of long range. In fact, the range is of the same order as the system size. In view of applications to cold atomic gases, a physically more relevant limit would be the Gross-Pitaevskii limit, ${ }^{26-28,39}$ where the interaction potential takes the form

$$
v(x)=N^{2} w(N x)
$$


for some fixed, $N$-independent function $w$. As discussed in more detail in Ref. 17, one expects that in this limit the excitation spectrum is still of the form (51), but with $\widehat{v}(p)$ replaced by $8 \pi a$, where $a$ denotes the scattering length of $w$.

- An even more challenging problem concerns the low energy excitation spectrum in the thermodynamic limit, and to study its relation to the property of superfluidity. There are no rigorous results available up to now, not even rough bounds are known. In fact, not even the absence of a spectral gap in the thermodynamic limit of an interacting Bose gas is rigorously known. We refer to Ref. 6 for further discussion of this topic.

- Also in the Hartree limit discussed in Sec. VII there are interesting open problems. One of them concerns the existence of collective excitations which should be described by solutions of the Hartree equation

$$
-\Delta \phi(x)+V(x)|\phi(x)|^{2}+v *|\phi|^{2}(x) \phi(x)=\mu \phi(x)
$$

for some $\mu \in \mathbb{R}$ which are different from $\phi_{0}$ and hence correspond to (non-linear) excited states of the Hartree functional. In the translation invariant case, collective excitations are related to the ground state via a Galileo transformation, as explained in Sec. VII A. In the absence of translation invariance, there is no such symmetry, and the existence of such states is therefore an open problem in general.

Moreover, the results in Refs. 9, 17,24, and 38 are all limited to the case where the Hartree functional (97) has a unique minimizer (up to a constant phase). However, at least in the case of attractive interactions, uniqueness will not hold, in general. Even with repulsive interactions, uniqueness can fail in the presence of magnetic fields or, equivalently, the case of rotating Bose gases. ${ }^{35,36}$ In this case, there can even be uncountably many minimizers. This happens, for instance, in rotating systems if the system is rotation invariant with respect to the axis of rotation, and the rotation speed is large enough for quantized vortices to form. If there is more than one such vortex, the rotation symmetry is necessarily broken in the minimizer, and hence there are infinitely many minimizers, which are all related via rotation. It would be nice to extend the results about the excitation spectrum in the Hartree limit to the case of multiple Hartree minimizers.

Note added in proof: For recent progress in this direction, see Ref. 47.

\section{ACKNOWLEDGMENTS}

Partial financial support by the NSERC is gratefully acknowledged.

${ }^{1}$ M. H. Anderson, J. R. Ensher, M. R. Matthews, C. E. Wieman, and E. A. Cornell, "Observation of Bose-Einstein condensation in a dilute atomic vapor," Science 269, 198-201 (1995).

${ }^{2}$ I. Bloch, J. Dalibard, and W. Zwerger, "Many-body physics with ultracold gases," Rev. Mod. Phys. 80, 885 (2008).

${ }^{3}$ N. Bogoliubov, "On the theory of superfluidity," J. Phys. (U.S.S.R.) 11, 23-32 (1947).

${ }^{4}$ S. N. Bose, "Plancks Gesetz und Lichtquantenhypothese," Z. Phys. 26, 178-181 (1924).

${ }^{5}$ N. R. Cooper, "Rapidly rotating atomic gases," Adv. Phys. 57, 539-616 (2008).

${ }^{6}$ H. D. Cornean, J. Dereziński, and P. Ziń, "On the infimum of the energy-momentum spectrum of a homogeneous Bose gas,” J. Math. Phys. 50, 062103 (2009).

${ }^{7}$ F. Dalfovo, S. Giorgini, L. P. Pitaevskii, and S. Stringari, "Theory of Bose-Einstein condensation in trapped gases," Rev. Mod. Phys. 71, 463-512 (1999).

${ }^{8}$ K. B. Davis, M. O. Mewes, M. R. Andrews, N. J. van Druten, D. S. Durfee, D. M. Kurn, and W. Ketterle, "Bose-Einstein condensation in a gas of sodium atoms," Phys. Rev. Lett. 75, 3969-3973 (1995).

${ }^{9}$ J. Dereziński and M. Napiórkowski, "Excitation spectrum of interacting bosons in the mean-field infinite-volume limit," Annales Henri Poincaré (2014) (published online); preprint arXiv:1305.3641.

${ }^{10}$ F. J. Dyson, "Ground-state energy of a hard-sphere gas," Phys. Rev. 106, 20-26 (1957).

${ }^{11}$ F. J. Dyson, E. H. Lieb, and B. Simon, "Phase transitions in quantum spin systems with isotropic and nonisotropic interactions," J. Stat. Phys. 18, 335-383 (1978).

${ }^{12}$ A. Einstein, "Quantentheorie des einatomigen idealen Gases," Sitzger. Preuss. Akad. Wiss., phys.-math. Kl. 261-267 (1924); 3-14 (1925)

${ }^{13}$ A. L. Fetter, "Rotating trapped Bose-Einstein condensates," Rev. Mod. Phys. 81, 647 (2009).

${ }^{14}$ L. L. Foldy, "Charged Boson gas,” Phys. Rev. 124, 649-651 (1961); 125, 2208 (1962) (Erratum). 
${ }^{15}$ J. Ginibre, "On the asymptotic exactness of the Bogoliubov approximation for many boson systems," Commun. Math. Phys. 8, 26-51 (1968).

${ }^{16}$ A. Giuliani and R. Seiringer, "The ground state energy of the weakly interacting Bose gas at high density," J. Stat. Phys. 135, 915-934 (2009).

${ }^{17}$ P. Grech and R. Seiringer, "The excitation spectrum for weakly interacting bosons in a trap," Commun. Math. Phys. 322, 559-591 (2013).

${ }^{18}$ C. Hainzl and R. Seiringer, "The BCS critical temperature for potentials with negative scattering length," Lett. Math. Phys. 84, 99-107 (2008).

${ }^{19}$ T. Kennedy, E. H. Lieb, and B. S. Shastry, "The XY model has long-range order for all spins and all dimensions greater than one," Phys. Rev. Lett. 61, 2582-2584 (1988).

${ }^{20}$ J. O. Lee, "Ground state energy of dilute Bose gas in small negative potential case," J. Stat. Phys. 134, 1-18 (2009).

${ }^{21}$ T. D. Lee and C. N. Yang, "Many body problem in quantum mechanics and quantum statistical mechanics," Phys. Rev. 105, 1119-1120 (1957); T. D. Lee, K. Huang, and C. N. Yang, "Eigenvalues and eigenfunctions of a Bose system of hard spheres and its low-temperature properties," ibid. 106, 1135-1145 (1957).

${ }^{22}$ M. Lewin, P. T. Nam, and N. Rougerie, "Derivation of Hartree's theory for generic mean-field Bose systems," Adv. Math. 254, 570-621 (2014); preprint arXiv:1303.0981.

${ }^{23}$ M. Lewin, P. T. Nam, and B. Schlein, "Fluctuations around Hartree states in the mean-field regime," preprint arXiv:1307.0665.

${ }^{24}$ M. Lewin, P. T. Nam, S. Serfaty, and J. P. Solovej, "Bogoliubov spectrum of interacting Bose gases," Commun. Pure Appl. Math. (2014) (published online); preprint arXiv:1211.2778.

${ }^{25}$ E. H. Lieb and W. Liniger, "Exact analysis of an interacting Bose gas. I. The general solution and the ground state," Phys. Rev. 130, 1605-1616 (1963); E. H. Lieb, "Exact analysis of an interacting Bose gas. II. The excitation spectrum," ibid. 130, 1616-1624 (1963).

${ }^{26}$ E. H. Lieb and R. Seiringer, "Proof of Bose-Einstein condensation for dilute trapped gases," Phys. Rev. Lett. 88, 170409 (2002).

${ }^{27}$ E. H. Lieb, R. Seiringer, J. P. Solovej, and J. Yngvason, The Mathematics of the Bose Gas and its Condensation, Oberwolfach Seminars Vol. 34 (Birkhäuser, 2005); e-print arXiv:cond-mat/0610117.

${ }^{28}$ E. H. Lieb, R. Seiringer, and J. Yngvason, "Bosons in a trap: A rigorous derivation of the Gross-Pitaevskii energy functional," Phys. Rev. A 61, 043602 (2000).

${ }^{29}$ E. H. Lieb, R. Seiringer, and J. Yngvason, "Justification of $c$-number substitutions in bosonic Hamiltonians," Phys. Rev. Lett. 94, 080401 (2005).

${ }^{30}$ E. H. Lieb and J. P. Solovej, "Ground state energy of the one-component charged Bose gas," Commun. Math. Phys. 217, 127-163 (2001); 225, 219-221 (2002) (Erratum).

${ }^{31}$ E. H. Lieb and J. P. Solovej, "Ground state energy of the two-component charged Bose gas," Commun. Math. Phys. 252, 485-534 (2004).

${ }^{32}$ E. H. Lieb and J. P. Solovej (unpublished).

${ }^{33}$ E. H. Lieb and J. Yngvason, "Ground state energy of the low density Bose gas," Phys. Rev. Lett. 80, 2504-2507 (1998).

${ }^{34}$ B. Schlein, "Effective equations for quantum dynamics," preprint arXiv:1208.0185.

${ }^{35}$ R. Seiringer, "Gross-Pitaevskii theory of the rotating Bose gas," Commun. Math. Phys. 229, 491-509 (2002).

${ }^{36}$ R. Seiringer, "Ground state asymptotics of a dilute, rotating gas," J. Phys. A: Math. Gen. 36, 9755-9778 (2003).

${ }^{37}$ R. Seiringer, "Free energy of a dilute Bose gas: Lower bound," Commun. Math. Phys. 279, 595-636 (2008).

${ }^{38}$ R. Seiringer, "The excitation spectrum for weakly interacting bosons," Commun. Math. Phys. 306, 565-578 (2011).

${ }^{39}$ R. Seiringer, "Hot topics in cold gases - A mathematical physics perspective," Japan. J. Math. 8, 185-232 (2013).

${ }^{40}$ R. Seiringer and D. Ueltschi, "Rigorous upper bound on the critical temperature of dilute Bose gases," Phys. Rev. B 80, 014502 (2009).

${ }^{41}$ J. P. Solovej, "Upper bounds to the ground state energies of the one- and two-component charged Bose gases," Commun. Math. Phys. 266, 797-818 (2006).

${ }^{42}$ A. Sütő, "Equivalence of Bose-Einstein condensation and symmetry breaking," Phys. Rev. Lett. 94, 080402 (2005).

${ }^{43}$ H.-T. Yau and J. Yin, "The second order upper bound for the ground energy of a Bose gas," J. Stat. Phys. 136, 453-503 (2009).

${ }^{44}$ J. Yin, "The ground state energy of dilute Bose gas in potentials with positive scattering length," Commun. Math. Phys. 295, 1-27 (2010).

45 J. Yin, "Free energies of dilute Bose gases: Upper bound," J. Stat. Phys. 141, 683-726 (2010).

${ }^{46}$ V. A. Zagrebnov and J.-B. Bru, "The Bogoliubov model of weakly imperfect Bose gas," Phys. Rep. 350, $291-434$ (2001).

${ }^{47}$ P. T. Nam and R. Seiringer, e-print arXiv:1402.1153. 